\title{
TYPE II CLATHRATE HYDRATE FORMATION IN COMETARY ICE ANALOGS IN VACUO
}

\author{
D.F. BLAKE, L. ALLAMANDOLA, \\ S. SANDFORD, D. HUDGINS \\ MS 239-4 \\ NASA/Ames Research Center \\ Moffett Field, CA 94035- USA
}

\author{
F. FREUND \\ Dept. of Physics \\ San Jose State University \\ San Jose, CA 95192 \\ USA
}

\begin{abstract}
Clathrate Hydrates can be formed under high vacuum conditions by annealing vapor-deposited amorphous ices of the appropriate composition. When astrophysically significant $\mathrm{H}_{2} \mathrm{O}: \mathrm{CH}_{3} \mathrm{OH}$ ices are deposited and annealed, Type II Clathrate Hydrates are formed which can hold up to 6 mole \% large guest molecules such as methanol and 12 mole $\%$ small guest molecules such as $\mathrm{CO}_{2}$ and $\mathrm{CO}$. The solid state transformation of amorphous mixed molecular ice into crystalline clathrate hydrate and its sublimation at higher temperatures may serve to explain heretofore anomalous mechanical and gas release properties observed in cometary ices and laboratory ice analog experiments.
\end{abstract}

\section{Introduction}

Many researchers have called upon clathrate hydrates to explain the anomalous properties of mixed molecular ices in various astrophysical environments, in particular comets. ${ }^{1}$ However, there has never been a satisfactory explanation as to how clathrate hydrates could form under the pressure/temperature conditions extant in small icy bodies or in the vacuum of space. In fact, evidence has been presented for the formation of clathrate hydrates from vapor-deposited amorphous ices by indirect methods (i.e., IR spectroscopy) by Devlin and co-authors. ${ }^{2,3}$ More recently it has been shown by electron diffraction and direct imaging in an electron microscope that type II clathrate hydrates can also form under high vacuum conditions from astrophysically relevant amorphous ice precursors. ${ }^{4}$

\section{Experimental Results}

In the experiments described in (4), either $\mathrm{H}_{2} \mathrm{O}: \mathrm{CH}_{3} \mathrm{OH}(2: 1)$ or $\mathrm{H}_{2} \mathrm{O}: \mathrm{CH}_{3} \mathrm{OH}(20: 1)$ was vapor-deposited under high vacuum onto a thin carbon substrate at $85 \mathrm{~K}$ inside a Transmission Electron Microscope (TEM). The deposit remained amorphous until about $120 \mathrm{~K}$ when a solid state phase separation occurred, producing a type II clathrate hydrate of 
$\mathrm{CH}_{3} \mathrm{OH}$ and a second amorphous phase containing the molecules which would not fit into the clathrate structure. At higher temperatures $(\sim 145 \mathrm{~K})$ the amorphous grain boundary phase sublimed leaving a porous network of clathrate. At $\sim 150 \mathrm{~K}$, the clathrate hydrate decomposed leaving porous hexagonal ice which sublimed at slightly higher temperatures in the vacuum of the TEM. When similar ice compositions were vapor-deposited and annealed in a cryogenic infrared (IR) spectrometer, only small shifts in absorption maxima were seen at and above the clathrate crystallization temperature. However, when $1 \% \mathrm{CO}_{2}$ was added as a local probe, the $v_{3}$ asymmetrical stretching vibration of $\mathrm{CO}_{2}$ was seen to shift from $2340 \mathrm{~cm}^{-1}$ to $2346 \mathrm{~cm}^{-1}$ during clathrate crystallization. This shift is identical to that reported for $\mathrm{CO}_{2}$ contained within the small cages of type II clathrate hydrates. ${ }^{3}$

\section{Discussion and Conclusions}

The above solid state reactions have important ramifications with regard to the mechanical and gas release properties of cometary ices. First, the initial crystallization event would enclathrate some of the guest species present in the precursor amorphous ice, and sweep the remaining neutrals and radicals to grain boundaries where they would be free to recombine. The energy released by crystallization and by the recombination of radicals could cause the anomalous evolution of gases at low temperatures. If enough foreign molecules were present in the original amorphous ice, a microporosity could develop which would allow escape of gases from deep within the nucleus, independent of solid state diffusion processes. Second, type II clathrate hydrates, once formed, can hold up to 12 mole \% small molecules in the two small cage sites present for each large cage site in the structure. Thus, type II clathrate hydrates, once formed, could enclathrate small molecules which percolated up from the interior during warming and annealing of deeper ice strata. All of these molecules would then be released upon the decomposition of the clathrate hydrate. Third, the mechanical properties of the clathrate hydrate will undoubtedly be different than the precursor amorphous ice. It is quite possible that surface exfoliation and mass shedding may be influenced by the presence / absence of clathrate hydrate. Last, the observed shift in the $v_{3}$ asymmetrical stretching vibration of $\mathrm{CO}_{2}$ opens the possibility to remotely observe clathrate hydrates using the IR signatures of their guest species.

\section{References}

1. A. H. Delsemme and P. Swings, Ann. d'Ap. 15, 1 (1952). A. H. Delsemme and D. C. Miller, Planet. Space. Sci. 18, 709 (1970). A. H. Delsemme and D. C. Miller, Planet Space Sci. 18, 717 (1970). S. L. Miller, in Physics and Chemistry of Ice, E. Whalley et al., Eds. (Royal Society of Canada, Ottawa, 1973), pp. 42-50. J. I. Lunine and D. J. Stevenson, Astrophys. J. Suppl. Ser. 58, 493 (1985).

2. J.E. Bertie and J. P. Devlin, J. Chem. Phys. 78(10), 6340 (1983). H. H. Richardson, P. J. Wooldridge, J. P. Devlin, J. Chem. Phys. 83(9), 4387 (1985).

3. F. Fleyfel and J. P. Devlin, J. Phys. Chem. 95, 3811 (1991).

4. D. Blake, L. Allamandola, S. Sandford, D. Hudgins and F. Freund, Science, in press. 